\title{
A Multiplier Theorem for Herz-Type Hardy Spaces Associated with the Dunkl Transform
}

\begin{abstract}
A. Gasmi
Department of Mathematics, Faculty of Sciences, Taibah University, Universities Road, P.O. Box 344, Al Madinah Al Monawarah 30001, Saudi Arabia
\end{abstract}

Correspondence should be addressed to A. Gasmi; gasmi1975@yahoo.fr

Received 29 June 2013; Accepted 14 October 2013

Academic Editor: Julian López-Gómez

Copyright (C) 2013 A. Gasmi. This is an open access article distributed under the Creative Commons Attribution License, which permits unrestricted use, distribution, and reproduction in any medium, provided the original work is properly cited.

The main purpose of this paper is to establish a Hörmander multiplier theorem for Herz-type Hardy spaces associated with the Dunkl transform.

\section{Introduction}

Let $T_{m}(f)$ be a multiplier operator defined in terms of Fourier transforms by $T_{m}(f)=\mathscr{F}^{-1}(m \mathscr{F}(f))$ for suitable functions $f$. The multiplier theorem of Hörmander [1] gives a sufficient condition on $m$ for the operator $T_{m}$ to be bounded on $L^{p}\left(\mathbb{R}^{n}\right)$ whenever $1<p<\infty$, namely, that $m$ is a bounded $C^{\ell}$ function on $\mathbb{R}^{n} \backslash\{0\}$ satisfying the Hörmander condition $M(2, \ell)$ as follows:

$$
\left(\int_{R}^{2 R}\left|m^{(s)}(\xi)\right|^{2} d \xi\right)^{1 / 2} \leq C R^{(n+1) / 2-s}, \quad \forall R>0,
$$

where $\ell$ is the least integer greater than $n / 2$ and $s=0,1, \ldots, \ell$. In [2], the authors proved that if $m$ satisfies the Hörmander condition with $\ell>n(1 / p-1 / 2)$, then $T_{m}$ is bounded on the Hardy spaces $H^{p}\left(\mathbb{R}^{n}\right)$ with $0<p \leq 1$.

In [3], the authors considered the following multiplier operator which is associated with the Dunkl transform:

$$
T_{m}^{\alpha}(f)=\mathscr{F}_{\alpha}^{-1}\left(m \mathscr{F}_{\alpha}(f)\right),
$$

where $\mathscr{F}_{\alpha}$ designs the Dunkl transform and using Hörmander's technique proved the following theorem.

Theorem 1. Let $\ell$ be the least integer greater than $\alpha+1$ and let $m$ be a bounded $C^{\ell}$-function on $\mathbb{R} \backslash\{0\}$ which satisfies the Hörmander condition $M_{\alpha}(2, \ell)$ as follows:

$$
\left(\int_{R}^{2 R}\left|m^{(s)}(\xi)\right|^{2} d \mu_{\alpha}(\xi)\right)^{1 / 2} \leq C R^{\alpha+1-s}, \quad \forall R>0,
$$

where $C$ is a constant independent of $R$ and $s=0,1, \ldots, \ell$. Then, the multiplier operator associated with the Dunkl transform can be extended to a bounded operator from $L^{p}\left(\mu_{\alpha}\right)$ into itself for $1<p<\infty$, where $L^{p}\left(\mu_{\alpha}\right)$ is the Lebesgue space on $\mathbb{R}$ with respect to the following measure:

$$
\mu_{\alpha}(x)=\left(2^{\alpha+1} \Gamma(\alpha+1)\right)^{-1}|x|^{2 \alpha+1}, \quad\left(\alpha>-\frac{1}{2}\right) .
$$

The Hardy spaces associated with Herz spaces can be regarded as the local version at the origin of the classical Hardy spaces $H^{p}$ and they are good substitutes for $H^{p}$ when we study the boundedness of nontranslation invariant operators. To establish the boundedness of operators in hardy-type spaces on $\mathbb{R}^{n}$, one usually appeals to the atomic decomposition characterization of these spaces. In $[4,5]$, the authors studied the Herz-type Hardy spaces $H \dot{K}_{\alpha, 2}^{\beta, p}$ for the Dunkl operator in one-dimension and gave an atomic decomposition characterization of these spaces. The aim of this work is to prove the following Hörmander multiplier theorem on the spaces $H \dot{K}_{\alpha, 2}^{\beta, p}$.

Theorem 2. Let $0<p \leq 1, \beta=(1 / p)-(1 / 2)$, and $\ell$ be an integer greater than $2(\alpha+1) \beta$. If $m$ satisfies the Hörmander condition $M_{\alpha}(2, \ell)$, then the operator $T_{m}^{\alpha}$ is bounded on $H \dot{K}_{\alpha, 2}^{\beta, p}$.

The paper is organized as follows. In Section 2, we recall some results about harmonic analysis and Herz-type Hardy spaces associated with the Dunkl operator on $\mathbb{R}$. In Section 3, we give the proof of the main result of this work. Then, as 
an application, we obtain the boundedness of the generalized Hilbert transform on $H \dot{K}_{\alpha, 2}^{\beta, p}$.

Throughout this paper, let $S(\mathbb{R})$ be the usual Schwartz space and let $\mathscr{E}(\mathbb{R})$ be the space of $C^{\infty}$-functions on $\mathbb{R}$. We always use $C$ to denote a positive constant that is independent of the main parameters involved but whose value may differ from line to line. We use the shorter notation $\|f\|_{p, \alpha}$ instead of $\|f\|_{L^{p}\left(\mu_{\alpha}\right)}$.

\section{Preliminaries}

In this section, we recapitulate some results about harmonic analysis on Dunkl hypergroups and the Herz-type Hardy space and its atomic decomposition which will be used later. For details, the reader is referred to [6-8].

Let $\alpha>-1 / 2$. We consider the differential-difference operator introduced in [9] as follows:

$$
\begin{aligned}
\Lambda_{\alpha}(f)(x)= & \frac{d f}{d x}(x)+\frac{2 \alpha+1}{x} \\
& \cdot \frac{f(x)-f(-x)}{2}, \quad f \in \mathscr{E}(\mathbb{R}),
\end{aligned}
$$

and call it the Dunkl operator.

For $\lambda \in \mathbb{C}$, the following initial value problem:

$$
\Lambda_{\alpha}(f)(x)=\lambda f(x), \quad f(0)=1, x \in \mathbb{R},
$$

has a unique solution $E_{\alpha}(\lambda \cdot)$ (called the Dunkl kernel) given by

$$
E_{\alpha}(z)=j_{\alpha}(i z)+\frac{z}{2(\alpha+1)} j_{\alpha+1}(i z), \quad z \in \mathbb{C}
$$

where $j_{\alpha}$ is the normalized Bessel function of the first kind (with order $\alpha$ ) defined on $\mathbb{C}$ by

$$
j_{\alpha}(z)=\Gamma(\alpha+1) \sum_{n=0}^{\infty}(-1)^{n} \frac{(z / 2)^{2 n}}{n ! \Gamma(n+\alpha+1)} .
$$

The integral representation of $E_{\alpha}$ is given by

$$
E_{\alpha}(i \lambda x)=\frac{\Gamma(\alpha+1)}{\sqrt{\pi} \Gamma(\alpha+(1 / 2))} \int_{-1}^{1}(1-t)\left(1-t^{2}\right)^{\alpha-(1 / 2)} e^{-i \lambda x t} d t
$$

From which, we get

$$
\left|\partial_{x}^{n} E_{\alpha}(i \lambda x)\right| \leq|\lambda|^{n}, \quad \lambda, x \in \mathbb{R}, n \in \mathbb{N} .
$$

The Dunkl transform $\mathscr{F}_{\alpha}$, which was introduced by [10] and studied in [11], is defined for $f \in L^{1}\left(\mu_{\alpha}\right)$ by

$$
\mathscr{F}_{\alpha}(f)(x)=\int_{\mathbb{R}} E_{\alpha}(-i x y) f(y) d \mu_{\alpha}(y), \quad x \in \mathbb{R} .
$$

This transform satisfies the following properties.

(i) For all $f \in L^{1}\left(\mu_{\alpha}\right)$, we have

$$
\left\|\mathscr{F}_{\alpha}(f)\right\|_{\infty, \alpha} \leq\|f\|_{1, \alpha} .
$$

(ii) For all $f \in L^{1}\left(\mu_{\alpha}\right)$ such that $\mathscr{F}_{\alpha}(f) \in L^{1}\left(\mu_{\alpha}\right)$, we have the following inversion formula:

$$
\mathscr{F}_{\alpha}^{-1}(f)(x)=\mathscr{F}_{\alpha}(f)(-x), \quad \text { a.e. } x \in \mathbb{R} .
$$

(iii) For all $f \in \mathcal{S}(\mathbb{R})$,

$$
\mathscr{F}_{\alpha}\left(\Lambda_{\alpha} f\right)(x)=i x \mathscr{F}_{\alpha}(f)(x) .
$$

(iv) $\mathscr{F}_{\alpha}$ is a topological isomorphism from $\mathcal{S}(\mathbb{R})$ into itself.

(v) $\mathscr{F}_{\alpha}$ is an isometric isomorphism of $L^{2}\left(\mu_{\alpha}\right)$, and we have the following Parseval formula:

$$
\begin{gathered}
\int_{\mathbb{R}} f(x) \overline{g(x)} d \mu_{\alpha}(x)=\int_{\mathbb{R}} \mathscr{F}_{\alpha}(f)(x) \overline{\mathscr{F}_{\alpha}(g)(x)} d \mu_{\alpha}(x), \\
\left\|\mathscr{F}_{\alpha}(f)\right\|_{2, \alpha}=\|f\|_{2, \alpha} .
\end{gathered}
$$

The following lemma can be proved, similar to Lemma 7.25 , page 343 , in [12].

Lemma 3. Let $\ell$ be the least integer greater than $\alpha+1$. If $m$ satisfies the Hörmander condition $M_{\alpha}(2, \ell)$, then there is a constant $C$ independent of $m$, such that if $q=1$ or $s-\ell+\alpha+1<$ $(\alpha+1) / q \leq \alpha+1$, the following inequality holds:

$$
\int_{R}^{2 R}\left|m^{(s)}(\xi)\right|^{2 q} d \mu_{\alpha}(\xi) \leq C R^{2(\alpha+1)-2 q s}, \quad \forall R>0 .
$$

Furthermore, in case $s-\ell+\alpha+1<0$, then $|x|^{s}\left|m^{(s)}(x)\right| \leq C$ and $m^{(s)}$ is continuous on $\mathbb{R} \backslash\{0\}$.

Notation. For all $x, y, z \in \mathbb{R}$, we put

$$
W_{\alpha}(x, y, z)=\left[1-\sigma_{x, y, z}+\sigma_{z, x, y}+\sigma_{z, y, x}\right] \Delta_{\alpha}(|x|,|y|,|z|),
$$

where

$$
\sigma_{x, y, z}= \begin{cases}\frac{x^{2}+y^{2}-z^{2}}{2 x y}, & \text { if } x, y \in \mathbb{R} \backslash\{0\}, \\ 0, & \text { otherwise, }\end{cases}
$$

$$
\begin{gathered}
\Delta_{\alpha}(|x|,|y|,|z|) \\
= \begin{cases}d_{\alpha} \frac{\left[\left((|x|+|y|)^{2}-z^{2}\right)\left(z^{2}-(|x|-|y|)^{2}\right)\right]^{\alpha-1 / 2}}{|x y z|^{2 \alpha}} & \text { if }|z| \in A_{x, y} \\
0, & \text { otherwise }\end{cases} \\
d_{\alpha}=\frac{2^{1-\alpha}(\Gamma(\alpha+1))^{2}}{\sqrt{\pi} \Gamma(\alpha+1 / 2)}, \\
A_{x, y}=[|| x|-| y||,|x|+|y|] .
\end{gathered}
$$


The Dunkl translation operator $\tau_{x}, x \in \mathbb{R}$ is defined for a continuous function $f$ on $\mathbb{R}$ by

$$
\tau_{x} f(y)=\int_{\mathbb{R}} f(z) d \gamma_{x, y}(z), \quad y \in \mathbb{R}
$$

where $\gamma_{x, y}$ is the signed measures given by

$$
d \gamma_{x, y}(z)= \begin{cases}W_{\alpha}(x, y, z) d \mu_{\alpha}(z), & \text { if } x, y \in \mathbb{R} \backslash\{0\}, \\ d \delta_{x}(z), & \text { if } y=0, \\ d \delta_{y}(z), & \text { if } x=0 .\end{cases}
$$

The operator $\tau_{x}$ has the following properties.

(i) For $x, y \in \mathbb{R}$ and a continuous function $f$ on $\mathbb{R}$, we have

$$
\tau_{x}(f)(y)=\tau_{y}(f)(x)
$$

(ii) For all $x \in \mathbb{R}$, the operator $\tau_{x}$ can be extended to $L^{p}\left(\mu_{\alpha}\right)(p \geq 1)$, and for $f \in L^{p}\left(\mu_{\alpha}\right)$, we have

$$
\left\|\tau_{x}(f)\right\|_{p, \alpha} \leq 3\|f\|_{p, \alpha} .
$$

(iii) For all $x, \lambda \in \mathbb{R}$ and $f \in L^{1}\left(\mu_{\alpha}\right)$, we have

$$
\mathscr{F}_{\alpha}\left(\tau_{x}(f)\right)(\lambda)=E_{\alpha}(i \lambda x) \mathscr{F}_{\alpha}(f)(\lambda) .
$$

Let $p, q, r \in[1, \infty]$ such that $1 / p+1 / q=1 / r+1$. The convolution product of $f \in L^{p}\left(\mu_{\alpha}\right)$ and $g \in L^{q}\left(\mu_{\alpha}\right)$ is defined by

$$
f *_{\alpha} g(x)=\int_{\mathbb{R}} \tau_{x}(f)(-y) g(y) d \mu_{\alpha}(y), \quad \text { a.e. } x,
$$

and we have

$$
\left\|f *_{\alpha} g\right\|_{r, \alpha} \leq 3\|f\|_{p, \alpha}\|g\|_{q, \alpha} .
$$

If $f, g \in L^{1}\left(\mu_{\alpha}\right)$, then

$$
\mathscr{F}_{\alpha}\left(f *_{\alpha} g\right)=\mathscr{F}_{\alpha}(f) \mathscr{F}_{\alpha}(g) .
$$

Now, let us recall the definition of the Herz-type Hardy space and its atomic decomposition. For $N \in \mathbb{N}$ being sufficiently large, we denote by $F_{N}$ the subset of $S(\mathbb{R})$ constituted by all those $\phi \in S(\mathbb{R})$ such that $\operatorname{supp}(\phi) \subset[-1,1]$ and for all $m, n \in \mathbb{N}$ such that $m, n \leq N$, we have

$$
\rho_{m, n}(\phi)=\sup _{x \in \mathbb{R}}(1+|x|)^{m}\left|\Lambda_{\alpha}^{n} \phi(x)\right| \leq 1 .
$$

Moreover, the system of seminorms $\left\{\rho_{m, n}\right\}_{m, n \in \mathbb{N}}$ generates the topology of $S(\mathbb{R})$.

Let $f \in S^{\prime}(\mathbb{R})$. We define the $\alpha$-grand maximal function $G_{\alpha}(f)$ of $f$ by

$$
G_{\alpha}(f)(x)=\sup _{t>0, \phi \in F_{N}}\left|\phi_{t} *_{\alpha} f(x)\right|, \quad x \in \mathbb{R},
$$

where $\phi_{t}$ is the dilation of $\phi$ given by

$$
\phi_{t}(x)=t^{-2(\alpha+1)} \phi\left(\frac{x}{t}\right), \quad x \in \mathbb{R} .
$$

Definition 4. Let $\beta \in \mathbb{R}, p \in] 0, \infty[$, and $q \in[1, \infty]$.

(i) The homogeneous weighted Herz space $\dot{K}_{\alpha, q}^{\beta, p}$ is the space constituted by all functions $f \in L_{\text {loc }}^{q}\left(\mu_{\alpha}\right)$, such that

$$
\|f\|_{\dot{K}_{\alpha, q}^{\beta, p}}=\left[\sum_{k=-\infty}^{\infty} 2^{2(\alpha+1) \beta k p}\left\|f \chi_{k}\right\|_{q, \alpha}^{p}\right]^{1 / p}<\infty
$$

where $\chi_{k}$ is the characteristic function of $A_{k}=\{x \in$ $\left.\mathbb{R} / 2^{k-1} \leq|x| \leq 2^{k}\right\}$

(ii) The nonhomogeneous weighted Herz space $K_{\alpha, q}^{\beta, p}$ is defined, as usual, by $K_{\alpha, q}^{\beta, p}=L^{q}\left(\mu_{\alpha}\right) \cap \dot{K}_{\alpha, q}^{\beta, p}$. Moreover, $\|f\|_{K_{q, \alpha}^{\beta, p}}=\|f\|_{q, \alpha}+\|f\|_{\dot{K}_{\alpha, q}^{\beta, p}}$.

Note that $\dot{K}_{\alpha, q}^{0, q}=K_{\alpha, q}^{0, q}=L^{q}\left(\mu_{\alpha}\right)$.

Definition 5. Let $\beta \in \mathbb{R}, p \in] 0, \infty]$, and $q \in] 1, \infty]$. The Herz-type Hardy space $H \dot{K}_{\alpha, q}^{\beta, p}$ is the space of distributions $f \in S^{\prime}(\mathbb{R})$ such that $G_{\alpha}(f) \in \dot{K}_{\alpha, q}^{\beta, p}$. Moreover, we define

$$
\|f\|_{H \dot{K}_{\alpha, q}^{\beta, p}}=\left\|G_{\alpha}(f)\right\|_{\dot{K}_{\alpha, q}^{\beta, p}} .
$$

In the same way, we define the space $H K_{\alpha, q}^{\beta, p}$ for the nonhomogeneous case.

Definition 6. Let $q \in] 1, \infty]$ and $\beta \geq 1-1 / q$. A measurable function $a$ on $\mathbb{R}$ is called a (central) $(\beta, q, s)$-atom if it satisfies the following:

(i) $\operatorname{supp}(a) \subset[-r, r]$, for some $r>0$,

(ii) $\|a\|_{q, \alpha} \leq r^{-2(\alpha+1) \beta}$,

(iii) $\int_{\mathbb{R}} a(x) x^{k} d \mu_{\alpha}(x)=0, k=0,1, \ldots, s$, where $s=$ $[2(\alpha+1)(\beta-1+1 / q)]$ and $[\cdot]$ denotes the integer part function.

The following theorem is shown in [4].

Theorem 7. Let $0<p \leq 1<q \leq \infty$ and $\beta \geq 1-1 / q$. Then, $f \in H \dot{K}_{\alpha, q}^{\beta, p}$ if and only if, for all $j \in \mathbb{N} \backslash\{0\}$, there exist $a(\beta, q, s)$-atom $a_{j}$ and $\lambda_{j} \in \mathbb{C}$, such that $\sum_{j=1}^{\infty}\left|\lambda_{j}\right|^{p}<\infty$ and $f=\sum_{j=1}^{\infty} \lambda_{j} a_{j}$. Moreover,

$$
\|f\|_{H \dot{K}_{\alpha, q}^{\beta, p}} \sim \inf \left(\sum_{j=1}^{\infty}\left|\lambda_{j}\right|^{p}\right)^{1 / p}
$$

where the infimum is taking over all atomic decompositions of $f$.

In the sequel, fix $q=2$ and $\beta=1 / p-1 / 2$. 
Definition 8. For $0<p \leq 1$. Set $s \geq[2(\alpha+1)(1 / p-1)], \varepsilon>$ $s / 2(\alpha+1), a=1-\frac{1}{p}+\varepsilon$, and $b=1 / 2+\varepsilon$. A central $(p, s, \varepsilon)-$ molecule is a function $M \in L^{2}\left(\mu_{\alpha}\right)$ satisfying the following:

(i) $M(x)|x|^{2(\alpha+1) b} \in L^{2}\left(\mu_{\alpha}\right)$,

(ii) $\|M\|_{2, \alpha}^{a / b}\left\|M(x)|x|^{2(\alpha+1) b}\right\|_{2, \alpha}^{1-a / b} \equiv N(M)<\infty$,

(iii) $\int_{\mathbb{R}} M(x) x^{k} d \mu_{\alpha}(x)=0, k=0,1, \ldots, s$.

Proposition 9. Let $(p, s, \varepsilon)$ be the triple cited in the previous definition. Every central $(p, s, \varepsilon)$-molecule $M$ belongs to $H \dot{K}_{\alpha, 2}^{\beta, p}$ and $\|M\|_{H \dot{K}_{\alpha, 2}^{\beta, p}} \leq C N(M)$, where the constant $C$ is independent of $M$.

Proof. Let $M$ be a central $(p, s, \varepsilon)$-molecule and suppose that $\|M\|_{H \dot{K}_{\alpha, 2}^{\beta, p}}=1$. In the general case, letting $\widetilde{M}=M /\|M\|_{H \dot{K}_{\alpha, 2}^{\beta, p}}$, we have $\|\widetilde{M}\|_{H \dot{K}_{\alpha, 2}^{\beta, p}}=1$.

Let $E_{0}=\{|x| \leq 1\}, E_{k}=\left\{2^{k-1}<|x| \leq 2^{k}\right\}$, and $M_{k}=$ $M \chi_{k}, k=1,2,3, \ldots$, where $\chi_{k}$ is the characteristic function of $E_{k}$. For each $k$, there exists a unique polynomial $Q_{k}$, of degree at most $s$, such that if $P_{k}=Q_{k} \chi_{k}$; then

$$
\int_{\mathbb{R}}\left(M_{k}-P_{k}\right) x^{j} d \mu_{\alpha}(x)=0, \quad j=0,1, \ldots, s .
$$

Using some ideas in [2], we can show that each $\left(M_{k}-\right.$ $\left.P_{k}\right)$ is a multiple of a central $(\beta, 2, s)$-atom with a sequence of coefficients in $l^{p}$. We also show that the sum $\sum_{k=0}^{+\infty} P_{k}$ can be written as an infinite linear combination of central $(\beta, \infty, s)$ atom with a sequence of coefficients in $l^{p}$. Since a $(\beta, \infty, s)$ atom is also $(\beta, 2, s)$-atom, hence,

$$
M=\sum_{k=0}^{+\infty} M_{k}=\sum_{k=0}^{+\infty}\left(M_{k}-P_{k}\right)+\sum_{k=0}^{+\infty} P_{k}=\sum_{i=0}^{+\infty} \lambda_{i} a_{i}
$$

where $a_{i}$ is a central $(\beta, 2, s)$-atom and $\sum_{k=0}^{+\infty}\left|\lambda_{i}\right|^{p}<\infty$. It follows from Theorem 7 that $M \in H \dot{K}_{\alpha, 2}^{\beta, p}$ and $\|M\|_{H \dot{K}_{\alpha, 2}^{\beta, p}} \leq$ $C N(M)$.

The following Lemma plays an important role in the proof of the main result of this work.

Lemma 10. Let a be a $(\beta, 2, s)$-atom. For all integer $0 \leq k \leq s$ and every $1 \leq u \leq \infty$, there exists a constant $C$ independent of a, such that

(i) $\left|\left(\mathscr{F}_{\alpha}(a)\right)^{(k)}(y)\right| \leq C|y|^{s+1-k}\|a\|_{2, \alpha}^{A}$,

$A=1-\frac{1}{\beta}\left(\frac{1}{2}+\frac{s+1}{2(\alpha+1)}\right) \quad y \in \mathbb{R}$,

(ii) $\left\|\left(\left(\mathscr{F}_{\alpha}(a)\right)^{(k)}(y)\right)^{2}\right\|_{u^{\prime}, \alpha} \leq C\|a\|_{2, \alpha}^{2-(1 / \beta)((k / \alpha+1)+(1 / u))}$,

$\frac{1}{u}+\frac{1}{u^{\prime}}=1 \quad y \in \mathbb{R}$.
Proof. (i) Let $a$ be a $(\beta, 2, s)$-atom. Consider that $r>0$ such that $\operatorname{supp}(a) \subset[-r, r]$ and that $\|a\|_{2, \alpha} \leq r^{-2(\alpha+1) \beta}$. From (9), (iii) of Definition (19), and the estimate for the remainder in Taylors' formula, it follows that

$$
\begin{aligned}
& \left(\mathscr{F}_{\alpha}(a)\right)^{(k)}(y) \\
& =C_{\alpha} \int_{-1}^{1}\left((1-t)\left(1-t^{2}\right)^{\alpha-1 / 2} t^{k}\right. \\
& \quad \times \int_{-r}^{r}(i x)^{k}\left[\exp (i x y t)-\sum_{n=0}^{s-k} \frac{(i x y t)^{n}}{n !}\right] \\
& \left.\quad \times a(x) d \mu_{\alpha}(x)\right) d t \\
& \leq C|y|^{s+1-k} \int_{-r}^{r}|x|^{s+1}|a(x)| d \mu_{\alpha}(x) \\
& \leq C|y|^{s+1-k}\|a\|_{2, \alpha}\left[\int_{-r}^{r}|x|^{2(s+1)} d \mu_{\alpha}(x)\right]^{1 / 2} \\
& \leq C|y|^{s+1-k}\|a\|_{2, \alpha} r^{s+\alpha+2} .
\end{aligned}
$$

From (ii) of Definition (19), we obtain

$$
\begin{gathered}
\left|\left(\mathscr{F}_{\alpha}(a)\right)^{(k)}(y)\right| \leq C|y|^{s+1-k}\|a\|_{2, \alpha}^{A}, \\
A=1-\frac{1}{\beta}\left(\frac{1}{2}+\frac{s+1}{2(\alpha+1)}\right) .
\end{gathered}
$$

(ii) For $u=1$,

$$
\left(\mathscr{F}_{\alpha}(a)\right)^{(k)}(y)=\int_{-r}^{r} \partial_{y}^{k} E_{\alpha}(-i y x) a(x) d \mu_{\alpha}(x) .
$$

Using (10), we get the following for all $y \in \mathbb{R}$ :

$$
\begin{aligned}
\left|\left(\mathscr{F}_{\alpha}(a)\right)^{(k)}(y)\right| \leq & C \int_{-r}^{r}|x|^{k}|a(x)| d \mu_{\alpha}(x) \\
\leq & C\left(\int_{-r}^{r}|a(x)|^{2} d \mu_{\alpha}(x)\right)^{1 / 2} \\
& \times\left(\int_{-r}^{r}|x|^{2 k} d \mu_{\alpha}(x)\right)^{1 / 2} \\
\leq & C\|a\|_{2, \alpha} r^{k+\alpha+1} .
\end{aligned}
$$

From (ii) of Definition (19), we obtain the following for all $y \in \mathbb{R}$ :

$$
\left|\left(\mathscr{F}_{\alpha}(a)\right)^{(k)}(y)\right|^{2} \leq C\|a\|_{2, \alpha}^{2-(1 / \beta)(k /(\alpha+1)+1)} .
$$

For $u=\infty$,

$$
\begin{aligned}
\int_{\mathbb{R}}\left|\left(\mathscr{F}_{\alpha}(a)\right)^{(k)}(x)\right|^{2} d \mu_{\alpha}(x) & \leq C \int_{-r}^{r}|x|^{2 k}|a(x)|^{2} d \mu_{\alpha}(x) \\
& \leq C r^{2 k}\|a\|_{2, \alpha}^{2} \\
& \leq C\|a\|_{2, \alpha}^{2-(1 / \beta)(k /(\alpha+1))} .
\end{aligned}
$$


For $1<u<\infty$,

$$
\begin{aligned}
& \int_{\mathbb{R}}\left|\left(\mathscr{F}_{\alpha}(a)\right)^{(k)}(y)\right|^{2 u^{\prime}} d \mu_{\alpha}(x) \\
& =\int_{\mathbb{R}}\left|\left(\mathscr{F}_{\alpha}(a)\right)^{(k)}(y)\right|^{2}\left|\left(\mathscr{F}_{\alpha}(a)\right)^{(k)}(y)\right|^{2 u^{\prime}-2} d \mu_{\alpha}(x) \\
& \leq C\|a\|_{2, \alpha}^{\left(u^{\prime}-1\right)(2-(1 / \beta)(k /(\alpha+1))} \int_{\mathbb{R}}\left|\left(\mathscr{F}_{\alpha}(a)\right)^{(k)}(x)\right|^{2} d \mu_{\alpha}(x) \\
& \leq C\|a\|_{2, \alpha}^{u^{\prime}\left(2-(1 / \beta)\left(k /(\alpha+1)+\left(1 / u^{\prime}\right)\right)\right.} .
\end{aligned}
$$

Finally, we get the following for all $y \in \mathbb{R}$ :

$$
\left\|\left(\left(\mathscr{F}_{\alpha}(a)\right)^{(k)}(y)\right)^{2}\right\|_{u^{\prime}, \alpha} \leq C\|a\|_{2, \alpha}^{2-(1 / \beta)(k /(\alpha+1)+(1 / u))} .
$$

\section{Proof of Theorem 2}

Let $0<p \leq 1$ and $\ell$ be an integer greater than $2(\alpha+1) \beta$. Set $s=[2(\alpha+1)(1 / p-1)], \epsilon=\ell / 2(\alpha+1)-(1 / 2), a=1-(1 / p)+$ $\epsilon$, and $b=\epsilon+(1 / 2)$.

We have $\ell-1 \geq s$; then, according to Proposition 9 to prove Theorem 2 it suffices to prove that, for any $(\beta, 2, \ell)$ atom $f, T_{m}^{\alpha} f$ is a central $(p, s, \epsilon)$-molecule with $N\left(T_{m}^{\alpha} f\right)<C$ for some constant $C$ independent of $f$. In other words, we need to check that

$$
\begin{aligned}
& \text { (i) } T_{m}^{\alpha} f(\xi), T_{m}^{\alpha} f(\xi)|\xi|^{\ell} \in L^{2}\left(\mu_{\alpha}\right), \\
& \text { (ii) }\left\|T_{m}^{\alpha} f\right\|_{2, \alpha}^{a / b}\left\|T_{m}^{\alpha} f(\xi)|\xi|^{\ell}\right\|_{2, \alpha}^{1-a / b} \equiv N\left(T_{m}^{\alpha} f\right)<C, \\
& \text { (iii) } \int_{\mathbb{R}} T_{m}^{\alpha} f(\xi) \xi^{j} d \mu_{\alpha}(\xi)=0 \quad \forall j=0,1, \ldots, s .
\end{aligned}
$$

Firstly, we prove (i) and (ii).

$m$ satisfies the Hörmander condition $M_{\alpha}(2, \ell)$; then, by Theorem 1 , there exists a constant $C$ independent of $f$, such that

$$
\left\|T_{m}^{\alpha} f\right\|_{2, \alpha} \leq C\|f\|_{2, \alpha} .
$$

From (14) and (13), we have

$$
\Lambda_{\alpha}^{\ell}\left(\mathscr{F}_{\alpha}\left(T_{m}^{\alpha} f\right)\right)(\xi)=\mathscr{F}_{\alpha}\left((-i x)^{\ell} T_{m}^{\alpha} f(x)\right)(\xi) .
$$

Then, by Plancherel theorem to estimate $\left\|T_{m}^{\alpha} f(\xi)|\xi|^{\ell}\right\|_{2, \alpha}$, it suffices to estimate $\left\|\Lambda_{\alpha}^{\ell}\left(m \mathscr{F}_{\alpha}(f)\right)\right\|_{2, \alpha}$, which turns out to prove that

$$
\left\|\Lambda_{\alpha}^{\ell}\left(m \mathscr{F}_{\alpha}(f)\right)\right\|_{2, \alpha} \leq C\|f\|_{2, \alpha}^{1-(\ell / 2(\alpha+1) \beta)} .
$$

By induction, we have

$$
\begin{aligned}
\Lambda_{\alpha}^{\ell}\left(m \mathscr{F}_{\alpha}(f)\right)(\xi)= & \sum_{r=0}^{l} a_{r} \xi^{r-\ell}\left(m \mathscr{F}_{\alpha}(f)\right)^{(r)}(\xi) \\
& +\sum_{r=0}^{l} b_{r} \xi^{r-\ell}\left(m \mathscr{F}_{\alpha}(f)\right)^{(r)}(-\xi)
\end{aligned}
$$

where $a_{r}$ and $b_{r}$ are constants.
But, using Leibniz formula, we have the following for $r \epsilon$ $\{0,1, \ldots, \ell\}$ :

$$
\left(m \mathscr{F}_{\alpha}(f)\right)^{(r)}(\xi)=\sum_{k=0}^{r} C_{r}^{k}\left(\mathscr{F}_{\alpha}(f)\right)^{(k)}(\xi)(m)^{(r-k)}(\xi) .
$$

So, to establish (47), it suffices to claim that

$$
\begin{aligned}
& \left\|\xi^{r-\ell}\left(\mathscr{F}_{\alpha}(f)\right)^{(k)}(\xi)(m)^{(r-k)}(\xi)\right\|_{2, \alpha} \\
& \quad \leq C\|f\|_{2, \alpha}^{1-(\ell / 2(\alpha+1) \beta)} \quad \text { for all integers } 0 \leq k \leq r \leq \ell .
\end{aligned}
$$

For the case $k=\ell$, we use Lemma 10 (ii) with $u=\infty$ and Lemma 3 to get the following:

$$
\begin{aligned}
\left\|\xi^{r-\ell}\left(\mathscr{F}_{\alpha}(f)\right)^{(k)}(\xi)(m)^{(r-k)}(\xi)\right\|_{2, \alpha} & \leq C\left\|\left(\mathscr{F}_{\alpha}(f)\right)^{(k)}\right\|_{2, \alpha} \\
& \leq C\|f\|_{2, \alpha}^{1-(\ell / 2(\alpha+1) \beta)} .
\end{aligned}
$$

For $0 \leq k<\ell$, we have

$$
\begin{aligned}
& \left\|\xi^{r-\ell}\left(\mathscr{F}_{\alpha}(f)\right)^{(k)}(\xi)(m)^{(r-k)}(\xi)\right\|_{2, \alpha}^{2} \\
& =\sum_{j \in \mathbb{Z}} \int_{2^{j}<|\xi|<2^{j+1}}|\xi|^{2(r-\ell)}\left|\left(\mathscr{F}_{\alpha}(f)\right)^{(k)}(\xi)\right|^{2} \\
& \quad \times\left|(m)^{(r-k)}(\xi)\right|^{2} d \mu_{\alpha}(\xi) \\
& =S_{1}+S_{2},
\end{aligned}
$$

where

$$
\begin{array}{r}
S_{1}=\sum_{j=-\infty}^{j_{0}} \int_{2^{j}<|\xi|<2^{j+1}}|\xi|^{2(r-\ell)}\left|\left(\mathscr{F}_{\alpha}(f)\right)^{(k)}(\xi)\right|^{2} \\
\times\left|(m)^{(r-k)}(\xi)\right|^{2} d \mu_{\alpha}(\xi), \\
S_{2}=\sum_{j=j_{0}+1}^{+\infty} \int_{2^{j}<|\xi|<2^{j+1}}|\xi|^{2(r-\ell)}\left|\left(\mathscr{F}_{\alpha}(f)\right)^{(k)}(\xi)\right|^{2} \\
\times\left|(m)^{(r-k)}(\xi)\right|^{2} d \mu_{\alpha}(\xi),
\end{array}
$$

and $j_{0}$ is the integer, such that

$$
2^{2(\alpha+1) j_{0}}<\|f\|_{2, \alpha}^{1 / \beta} \leq 2^{2(\alpha+1)\left(j_{0}+1\right)} .
$$

Firstly, we estimate $S_{1}$.

Using (i) of Lemma 10 and the fact that $m$ satisfies the Hörmander condition $M_{\alpha}(2, \ell)$, we get

$$
\begin{aligned}
& \int_{2^{j}<|\xi|<2^{j+1}}|\xi|^{2(r-\ell)}\left|\left(\mathscr{F}_{\alpha}(f)\right)^{(k)}(\xi)\right|^{2}\left|(m)^{(r-k)}(\xi)\right|^{2} d \mu_{\alpha}(\xi) \\
& \leq C\|f\|_{2, \alpha}^{2-(1 / \beta)(\ell /(\alpha+1)+1)} \\
& \quad \times \int_{2^{j}<|\xi|<2^{j+1}}|\xi|^{2(r+1-k)}\left|(m)^{(r-k)}(\xi)\right|^{2} d \mu_{\alpha}(\xi) \\
& \leq C\|f\|_{2, \alpha}^{2-(1 / \beta)(\ell /(\alpha+1)+1)} 2^{2(\alpha+1) j} .
\end{aligned}
$$


By (54), we obtain

$$
S_{1} \leq C\|f\|_{2, \alpha}^{2-(1 / \beta)(\ell /(\alpha+1)+1)} 2^{2(\alpha+1) j_{0}} \leq C\|f\|_{2, \alpha}^{2-(\ell /(\alpha+1) \beta)} .
$$

Now, we estimate $S_{2}$. By Holder's inequality, we have

$$
\begin{gathered}
\int_{2^{j}<|\xi|<2^{j+1}}|\xi|^{2(r-\ell)}\left|\left(\mathscr{F}_{\alpha}(f)\right)^{(k)}(\xi)\right|^{2}\left|(m)^{(r-k)}(\xi)\right|^{2} d \mu_{\alpha}(\xi) \\
\leq 2^{2 j(r-\ell)}\left(\int_{2^{j}<|\xi|<2^{j+1}}\left|\left(\mathscr{F}_{\alpha}(f)\right)^{(k)}(\xi)\right|^{2 u^{\prime}} d \mu_{\alpha}(\xi)\right)^{1 / u^{\prime}} \\
\quad \times\left(\int_{2^{j}<|\xi|<2^{j+1}}\left|(m)^{(r-k)}(\xi)\right|^{2 u} d \mu_{\alpha}(\xi)\right)^{1 / u} .
\end{gathered}
$$

Using (ii) of Lemmas 10 and 3, we get

$$
S_{2} \leq C\|f\|_{2, \alpha}^{2-(1 / \beta)((k /(\alpha+1))+(1 / u))} \sum_{j=j_{0}+1}^{+\infty}\left(2^{(2(\alpha+1) / u)-2(\ell-k)}\right)^{j} .
$$

To guarantee the convergence of this summation, we choose the pair $(k, u)$ as follows:

(a) if $l-k>\alpha+1$, we choose $u=1$;

(b) if $0<l-k \leq \alpha+1$ and $k>\alpha+1$, we choose $u=\infty$;

(c) if $0<l-k \leq \alpha+1$ and $k \leq \alpha+1$, we choose $0<u<\infty$ such that $k>(\alpha+1)(1-(1 / u))$.

Furthermore, by (54), we get

$$
\begin{aligned}
S_{2} & \leq C\|f\|_{2, \alpha}^{2-(1 / \beta)(k /(\alpha+1)+(1 / u))}\left(2^{(2(\alpha+1) / u)-2(\ell-k)}\right)^{j_{0}+1} \\
& \leq C\|f\|_{2, \alpha}^{2-(\ell /(\alpha+1) \beta)} .
\end{aligned}
$$

Finally, combining (56) and (59), we obtain (47). (i) and (ii) are hence proved.

To prove (iii), it suffices to prove that $T_{m}^{\alpha} f(\xi) \xi^{j} \in L^{1}\left(\mu_{\alpha}\right)$ for all integer $0 \leq j \leq s$ and $\Lambda_{\alpha}^{j}\left(m \mathscr{F}_{\alpha}(f)\right)(0)=0$ : indeed if $T_{m}^{\alpha} f(\xi) \xi^{j} \in L^{1}\left(\mu_{\alpha}\right)$ according to (14), which we have $\Lambda_{\alpha}^{j}\left(\mathscr{F}_{\alpha}\left(T_{m}^{\alpha} f\right)\right)(x)=C \mathscr{F}_{\alpha}\left(T_{m}^{\alpha} f(\xi) \xi^{j}\right)(x)$ is continuous, and hence $\int_{\mathbb{R}} T_{m}^{\alpha} f(\xi) \xi^{j} d \mu_{\alpha}(\xi)=C \Lambda_{\alpha}^{j}\left(m \mathscr{F}_{\alpha}(f)\right)(0)$.

Now, we check $T_{m}^{\alpha} f(\xi) \xi^{j} \in L^{1}\left(\mu_{\alpha}\right)$. We write $\int_{\mathbb{R}}\left|T_{m}^{\alpha} f(\xi) \xi^{j}\right| d \mu_{\alpha}(\xi)=I_{1}+I_{2}$, where

$$
\begin{gathered}
I_{1}=\int_{|\xi| \leq 1}\left|T_{m}^{\alpha} f(\xi) \xi^{j}\right| d \mu_{\alpha}(\xi), \\
I_{2}=\int_{|\xi|>1}\left|T_{m}^{\alpha} f(\xi) \xi^{j}\right| d \mu_{\alpha}(\xi) .
\end{gathered}
$$

Using the fact that $T_{m}^{\alpha} f \in L^{2}\left(\mu_{\alpha}\right)$ and Schwarz's inequality, we get

$$
\begin{aligned}
I_{1} & \leq \int_{|\xi| \leq 1}\left|T_{m}^{\alpha} f(\xi)\right| d \mu_{\alpha}(\xi) \\
& \leq\left(\int_{|\xi| \leq 1}\left|T_{m}^{\alpha} f(\xi)\right|^{2} d \mu_{\alpha}(\xi)\right)^{1 / 2}\left(\int_{|\xi| \leq 1} d \mu_{\alpha}(\xi)\right)^{1 / 2} \\
& \leq C\left\|T_{m}^{\alpha} f\right\|_{2, \alpha} \leq \infty
\end{aligned}
$$

For $0 \leq j \leq s$, we have

$$
\begin{aligned}
I_{2} \leq & \int_{|\xi|>1}\left|T_{m}^{\alpha} f(\xi) \xi^{s}\right| d \mu_{\alpha}(\xi) \\
\leq & \left(\int_{|\xi|>1}\left|T_{m}^{\alpha} f(\xi)\right|^{2}\left|\xi^{2 \ell}\right| d \mu_{\alpha}(\xi)\right)^{1 / 2} \\
& \times\left(\int_{|\xi|>1}\left|\xi^{2(s-\ell)}\right| d \mu_{\alpha}(\xi)\right)^{1 / 2} \\
= & \left\|T_{m}^{\alpha} f(\xi)|\xi|^{l}\right\|_{2, \alpha}\left(\int_{|\xi|>1}\left|\xi^{2(s-\ell)}\right| d \mu_{\alpha}(\xi)\right)^{1 / 2} .
\end{aligned}
$$

Using the fact that $s-\ell<\alpha+1$, we get $I_{2} \leq C$.

Finally, we check

$$
\Lambda_{\alpha}^{j}\left(m \mathscr{F}_{\alpha}(f)\right)(0)=0, \quad 0 \leq j \leq s .
$$

We have

$$
\begin{aligned}
\Lambda_{\alpha}^{j}\left(m \mathscr{F}_{\alpha}(f)\right)(h)= & \sum_{r=0}^{j} a_{r} h^{r-j}\left(m \mathscr{F}_{\alpha}(f)\right)^{(r)}(h) \\
& +\sum_{r=0}^{j} b_{r} h^{r-j}\left(m \mathscr{F}_{\alpha}(f)\right)^{(r)}(-h),
\end{aligned}
$$

where $a_{r}$ and $b_{r}$ are constants. Then, to prove (63), it suffices to prove that

$$
\begin{array}{r}
\lim _{h \rightarrow 0}\left|h^{r-j} m^{(r-k)}(h)\left(\mathscr{F}_{\alpha}(f)\right)^{(k)}(h)\right|=0, \\
\text { for all integers } 0 \leq k \leq r \leq j \leq s .
\end{array}
$$

By (i) of Lemma 10, we have

$$
\begin{aligned}
& \left|h^{r-j} m^{(r-k)}(h)\left(\mathscr{F}_{\alpha}(f)\right)^{(k)}(h)\right| \\
& \quad \leq C|h|^{s+1-j}|h|^{r-k}\left|m^{(r-k)}(h)\right|\|f\|_{2, \alpha}^{A} .
\end{aligned}
$$

According to Lemma 3, we have $|h|^{r-k}\left|m^{(r-k)}(h)\right| \leq C$; indeed $2(r-k-\ell)+\alpha+1<0$; then, we obtain

$$
\lim _{h \rightarrow 0}\left|h^{r-j} m^{(r-k)}(h)\left(\mathscr{F}_{\alpha}(f)\right)^{(k)}(h)\right| \leq C \lim _{h \rightarrow 0}|h|^{s+1-j}=0,
$$

where (63) is hence proved. This finishes the proof of Theorem 2.

Corollary 11. Let $0<p \leq 1$. Then, the generalized Hilbert transform $H_{\alpha}$ defined by

$$
H_{\alpha}(f)=\frac{\Gamma(\alpha+(3 / 2))}{\sqrt{\pi} \Gamma(\alpha+1)} \lim _{\varepsilon \rightarrow 0} \int_{|y|>\varepsilon} \frac{\tau_{x}(f)(-y)}{y} d y,
$$

where $\tau_{x}$ is given by (19), is bounded on $H \dot{K}_{\alpha, 2}^{\beta, p}$.

Proof. From Proposition 3.6 in [3], the generalized Hilbert transform $H_{\alpha}$ is a multiplier operator $T_{m}^{\alpha}$ with $m(\xi)=$ $-\operatorname{sign}(\xi)$; then the proof of the corollary follows from Theorem 2. 


\section{Acknowledgment}

This paper was supported by a generous grant from Taibah University Research Project.

\section{References}

[1] L. Hörmander, "Estimates for translation invariant operators in $L_{p}$ spaces," Acta Mathematica, vol. 104, pp. 93-140, 1960.

[2] M. H. Taibleson and G. Weiss, "The molecular characterization of certain Hardy spaces," Asterisque, vol. 77, pp. 67-149, 1980.

[3] B. Amri, A. Gasmi, and M. Sifi, "Linear and bilinear multiplier operators for the Dunkl transform," Mediterranean Journal of Mathematics, vol. 7, no. 4, pp. 503-521, 2010.

[4] A. Gasmi, M. Sifi, and F. Soltani, "Herz-type Hardy spaces for the Dunkl operator on the real line," Fractional Calculus \& Applied Analysis, vol. 9, no. 3, pp. 287-306, 2006.

[5] A. Gasmi and F. Soltani, "Convolution operators and BochnerRiesz means on Herz-type Hardy spaces in the Dunkl setting," International Journal of Mathematics and Mathematical Sciences, Article ID 204509, 17 pages, 2010.

[6] M. Rösler, "Bessel-type signed hypergroups on $\mathbb{R}$," in Probability measures on groups and related structures, XI (Oberwolfach, 1994), pp. 292-304, World Science Publisher, River Edge, NJ, USA, 1995.

[7] S. Thangavelu and $\mathrm{Y} . \mathrm{Xu}$, "Convolution operator and maximal function for the Dunkl transform," Journal d'Analyse Mathématique, vol. 97, no. 1, pp. 25-55, 2005.

[8] K. Trimèche, "Paley-Wiener theorems for the Dunkl transform and Dunkl translation operators," Integral Transforms and Special Functions, vol. 13, no. 1, pp. 17-38, 2002.

[9] C. F. Dunkl, "Differential-difference operators associated to reflection groups," Transactions of the American Mathematical Society, vol. 311, no. 1, pp. 167-183, 1989.

[10] C. F. Dunkl, "Hankel transforms associated to finite reflection groups," in Hypergeometric Functions on Domains of Positivity, Jack Polynomials, and Applications (Tampa, FL, 1991), vol. 138, American Mathematical Society, Providence, RI, USA, 1992.

[11] M. F. E. de Jeu, “The Dunkl transform," Inventiones Mathematicae, vol. 113, no. 1, pp. 147-162, 1993.

[12] J. García-Cuerva and J. L. Rubio de Francia, Weighted Norm Inequalities and Related Topics, vol. 116, North-Holland, Amsterdam, 1985. 


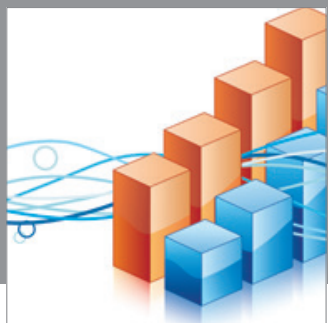

Advances in

Operations Research

mansans

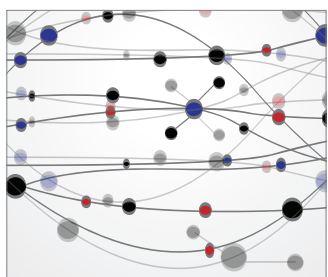

The Scientific World Journal
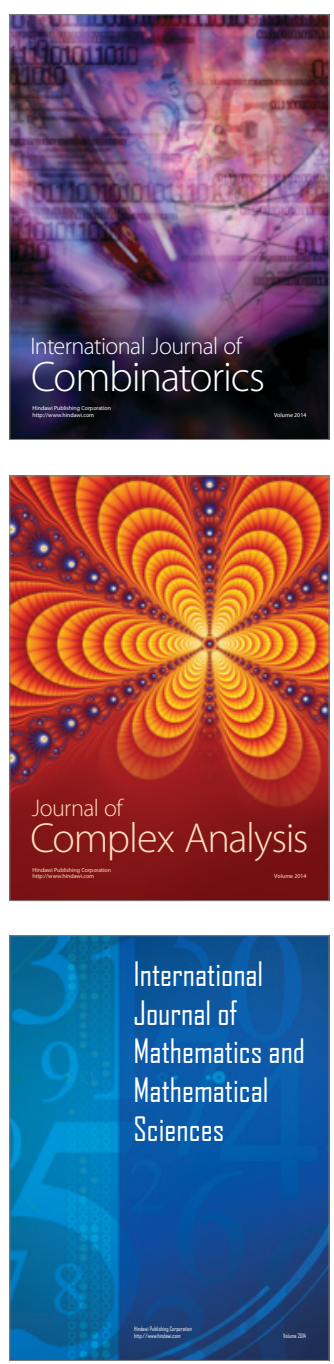
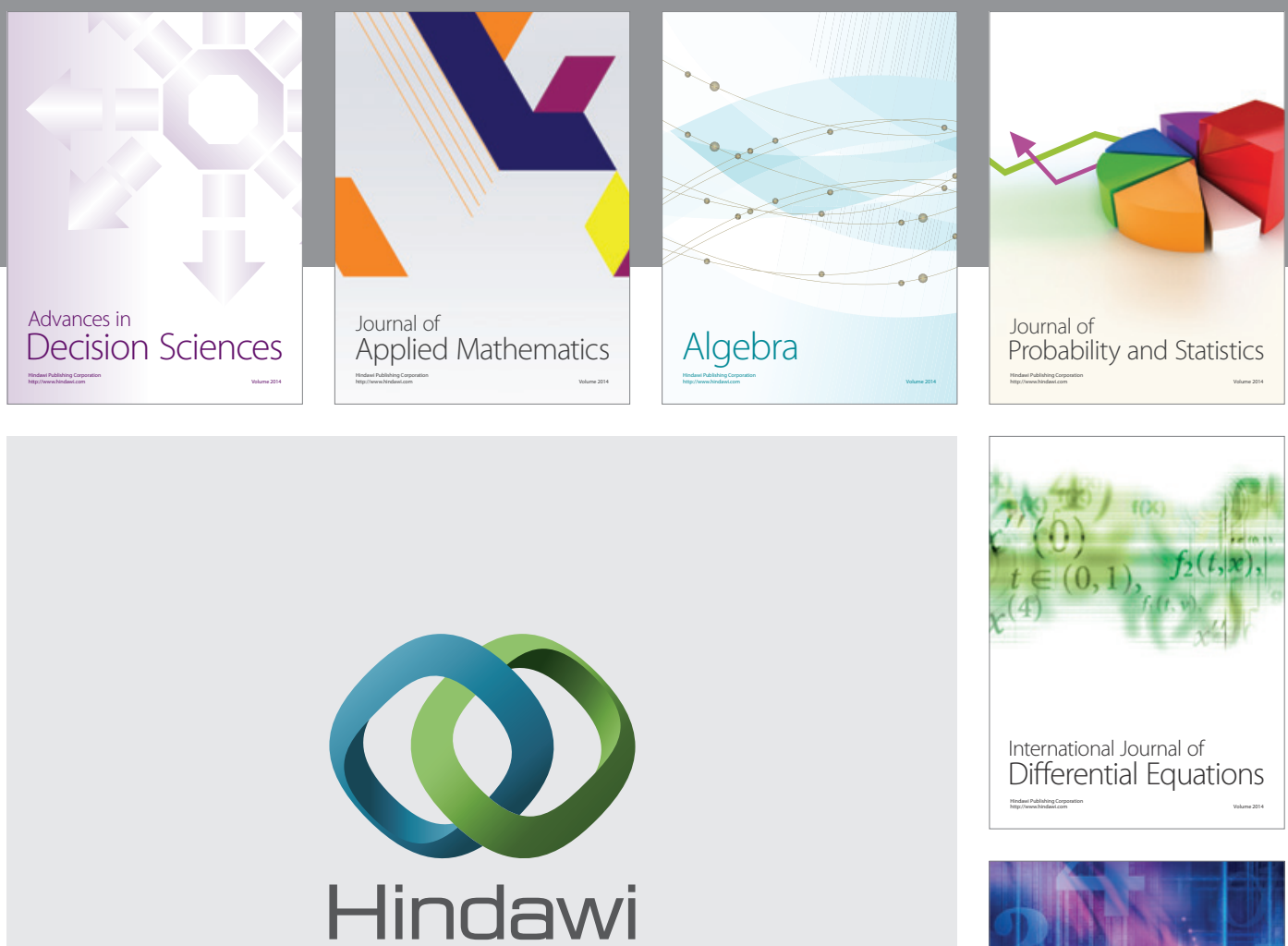

Submit your manuscripts at http://www.hindawi.com
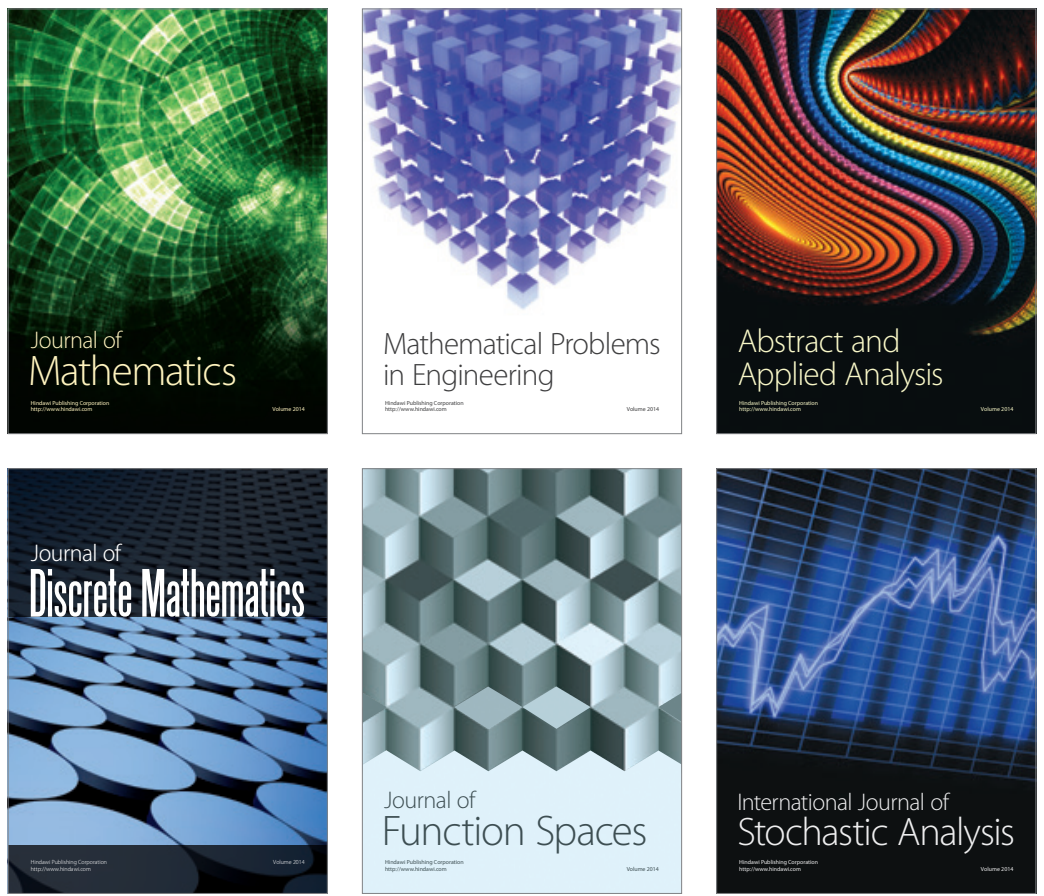

Journal of

Function Spaces

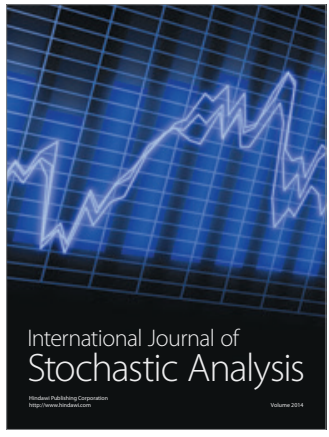

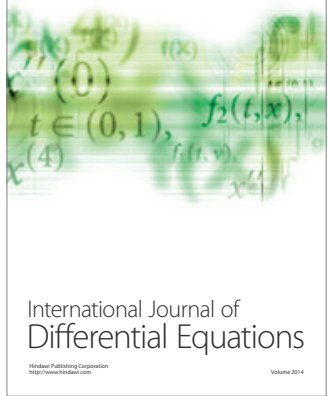
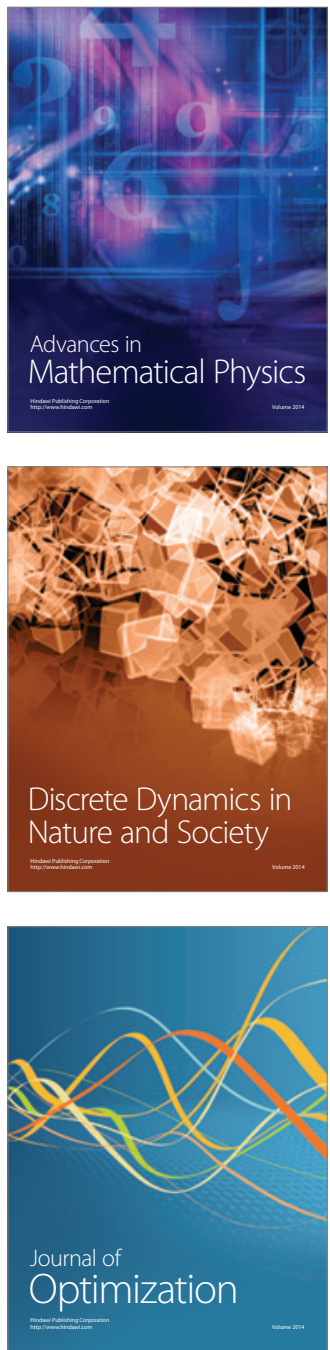